\title{
Circular asymmetry of the paleomagnetic directions observed at low latitude volcanic sites
}

\author{
Hidefumi Tanaka \\ Faculty of Education, Kochi University, Kochi 780-8520, Japan
}

(Received March 3, 1999; Revised October 1, 1999; Accepted October 12, 1999)

\begin{abstract}
The shape of the distribution in field directions and VGP positions was studied by Bingham statistics applied to a paleomagnetic dataset from lavas for the last 5 my by McElhinny and McFadden (1997), which includes those with VGP latitude higher than $45^{\circ}$. Data from Hawaii clearly show an oval shaped distribution of field directions, elongated along the meridian plane, while distribution of VGP is almost circular. Analysis on the global data divided by latitudinal bands also indicate more elongation in field directions for low latitude bands. This feature was interpreted as a general indication of dipole nature of the paleomagnetic field. For Hawaii data, however, possibility of the Pacific Nondipole Low is also suggested due to the large elongation parameter and very high significance of Fisher distribution to describe the VGP positions together with the small ASD. Although this elongated shape in the distribution of field directions is not clear for most of the individual site, the directions of the principal axes are sensitive enough to depict this feature at almost all sites.
\end{abstract}

\section{Introduction}

The shape of the distribution of field directions is distorted when paleomagnetic directions are mapped into virtual geomagnetic poles (VGPs). In the theory of mapping from field to pole or vice versa (Cox, 1970), the Fisher distribution (Fisher, 1953) was supposed for the original data set and the mapped distribution was approximated by an ellipse. Hence, if the observed paleomagnetic directions are in the form of Fisher distribution, the distribution of VGP has an elongated shape with the degree of elongation depending on the latitude of the observation site. On the other hand, if the dispersion of the paleomagnetic field is entirely due to random dipole wobble, so that the dipole positions follow the Fisher distribution, then this distribution will be faithfully reflected in the calculated VGP positions at all sites whereas the observed distribution of paleomagnetic directions will be elongated. Creer $e t$ al. (1959) first noted the elongated distribution of paleodirections, and a model of paleosecular variation (PSV) which entirely consists of a dipole wobble was proposed (Creer, 1962). Although the dipole wobble is not the only solution for circular distribution of VGPs (ex., Camps and Prévot, 1996), the shape of the data distribution is very important in the study of PSV. In spite of this fact, only a few authors paid attention to the shape of the data distribution in the studies of PSV from lavas (Baag and Helsley, 1974; Tsunakawa, 1988).

One of useful statistics to describe an elongated distribution of unit vectors was given by Bingham (1974). The density function of the Bingham distribution is given

Copy right $\odot$ The Society of Geomagnetism and Earth, Planetary and Space Sciences (SGEPSS); The Seismological Society of Japan; The Volcanological Society of Japan; The Geodetic Society of Japan; The Japanese Society for Planetary Sciences. as,

$$
b(\theta, \phi)=\frac{1}{4 \pi d\left(k_{1}, k_{2}\right)} e^{\left(k_{1} \cos ^{2} \phi+k_{2} \sin ^{2} \phi\right) \sin ^{2} \theta}
$$

where $\theta$ and $\phi$ are colatitude and longitude in spherical coordinate, $k_{1}$ and $k_{2}$ are concentration parameters $\left(k_{1} \leq k_{2}\right.$ $\leq 0)$, and $d\left(k_{1}, k_{2}\right)$ is a normalization constant. The data of $k_{1} \sim k_{2}$ indicate almost circular distribution while moderate difference of $k_{1}$ and $k_{2}$ suggests elongation in the distribution. Application of the Bingham statistics is easy if a numerical method is used (Onstott, 1980). First, an orientation matrix, $\boldsymbol{T}$, is defined from $N$ unit vectors as,

$$
\boldsymbol{T}=\left(\begin{array}{ccc}
\sum x_{i}^{2} & \sum x_{i} y_{i} & \sum x_{i} z_{i} \\
\sum x_{i} y_{i} & \sum y_{i}^{2} & \sum y_{i} z_{i} \\
\sum x_{i} z_{i} & \sum y_{i} z_{i} & \sum z_{i}^{2}
\end{array}\right)
$$

where summations are taken from 1 to $N$. Eigenvalues of $\boldsymbol{T}, \tau_{1}, \tau_{2}$, and $\tau_{3}\left(\tau_{1} \leq \tau_{2} \leq \tau_{3}, \tau_{1}+\tau_{2}+\tau_{3}=N\right)$, and corresponding eigenvectors, $\boldsymbol{t}_{1}, \boldsymbol{t}_{2}$, and $\boldsymbol{t}_{3}$, collectively give a general idea of the distribution. The maximum likelihood estimates of the Bingham's concentration parameters, $k_{1}$ and $k_{2}$, are given by maximizing the $\log$ likelihood function,

$$
F=-N \log (4 \pi)-N \log \left(d\left(k_{1}, k_{2}\right)\right)+k_{1} \tau_{1}+k_{2} \tau_{2}
$$

$95 \%$ confidence radii around the mean $\left(\boldsymbol{t}_{3}\right)$ is given by,

$$
\alpha_{31}=2.45 \sigma_{31}, \quad \alpha_{32}=2.45 \sigma_{32}
$$

where $\sigma_{i j}$ is given by, 


$$
\sigma_{i j}^{2}=\frac{1}{2\left(k_{i}-k_{j}\right)\left(\tau_{i}-\tau_{j}\right)} .
$$

I would like to point out here that the notation of $\alpha_{i j}$ given by Onstott (1980) is in error by a factor of $\sqrt{N}^{i j}$. According to Bingham (1974), $\alpha_{i j}$ given by Eq. (4) is correct. This was ascertained by a computer experiment in which a population following the Bingham distribution was generated (Appendix), and then the distribution of the sample means ( $\boldsymbol{t}_{3}$ vectors) were calculated. The radii of the axes of the oval which contains $95 \%$ of the sample means agreed with those given by Eq. (4).

\section{Simulation of Mapping between Field and VGP}

Computer simulations of mapping field directions to poles or vice versa were made to examine the shape of the distribution of the transferred data. The ratio of radii of the $95 \%$ confidence region,

$$
\alpha_{32} / \alpha_{31}
$$

was used as an elongation parameter, while angular standard deviation (ASD), $S$, was calculated by the usual expression,

(a)

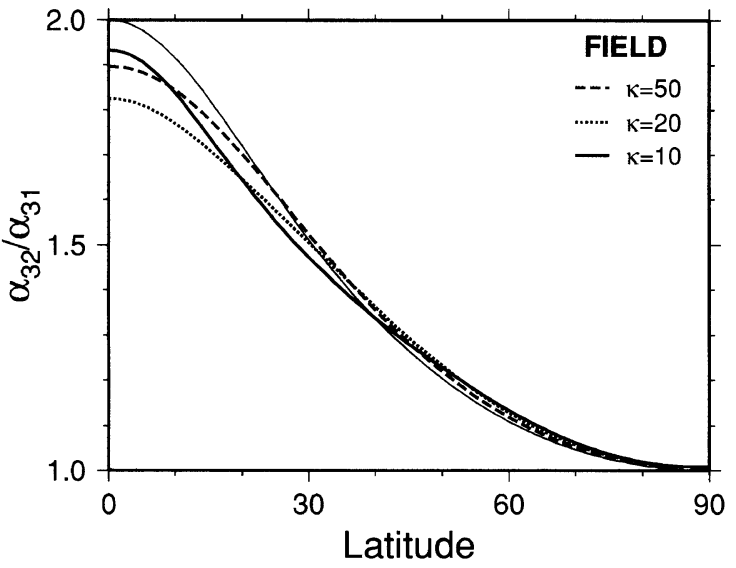

(b)

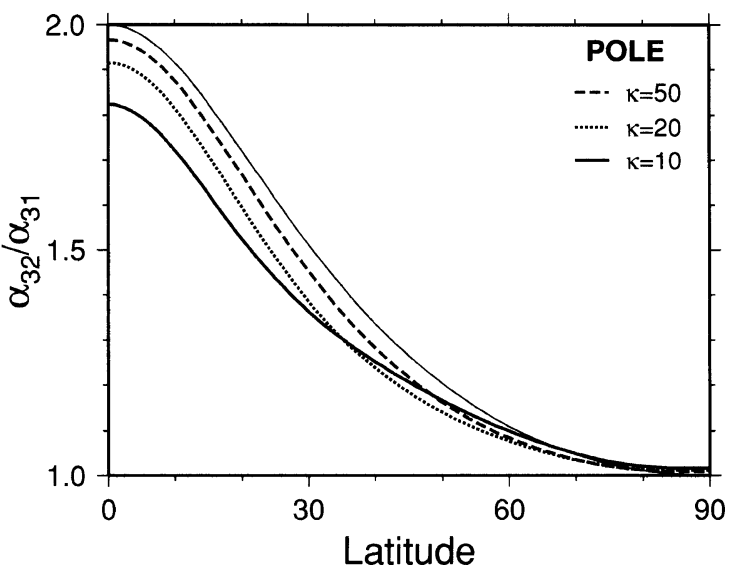

Fig. 1. Latitude variation of $\alpha_{32} / \alpha_{31}$ for three cases of precision parameter for field directions mapped from Fisherian poles (a) and VGP positions mapped from Fisherian field directions (b). Theoretical curves by Cox (1970) are shown by a fine solid line.

$$
S^{2}=\frac{1}{N-1} \sum_{i=1}^{N} \Delta_{i}^{2}
$$

where $\Delta_{i}$ is the angular distance of $i$-th vector from the mean. Latitude variations of such parameters were examined for several numbers of random points, from 1000 to 30000 , for three values of $\kappa(10,20$, and 50). Latitude variation curves for these parameters easily converged for the random numbers larger than 5000, and here the results are shown for the random numbers of 20000. Latitude variations of $\alpha_{32} / \alpha_{31}$ for field directions transferred from Fisherian poles and for VGP positions transferred from Fisherian field directions are shown in Figs. 1(a) and (b), respectively. The ratio of $\alpha_{32} / \alpha_{31}$ decreases from a value near 1.9 down to 1.0 as the latitude increases from equator to pole. Therefore, this parameter will be useful to discriminate which of field or pole is more Fisherian in the data of paleosecular variation. The variation curves for field and VGP are similar to those from theoretical prediction (Cox, 1970) which are included by fine solid curves in the figures. However, there are quite much discrepancies between simulation and theory especially at low latitude when $\kappa$ is not large. The latitude variation curve of $\alpha_{32} / \alpha_{31}$ for VGPs transferred from Fisherian field directions become closer to the theoretical

(a)

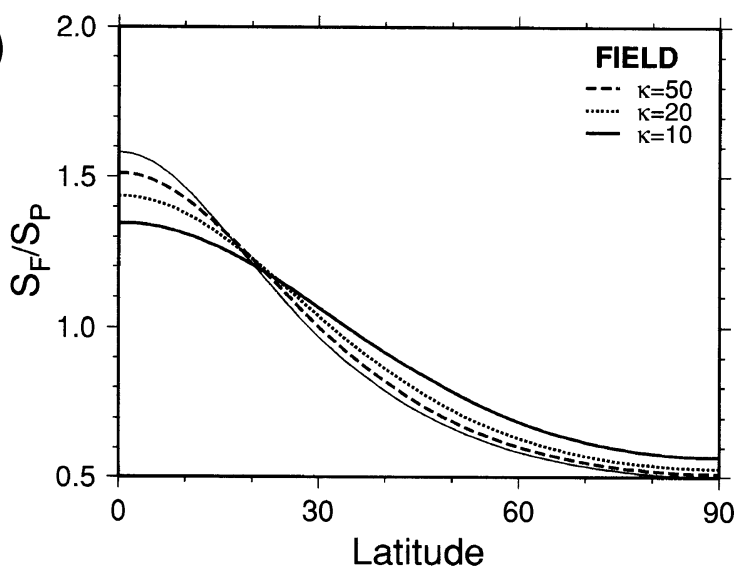

(b)

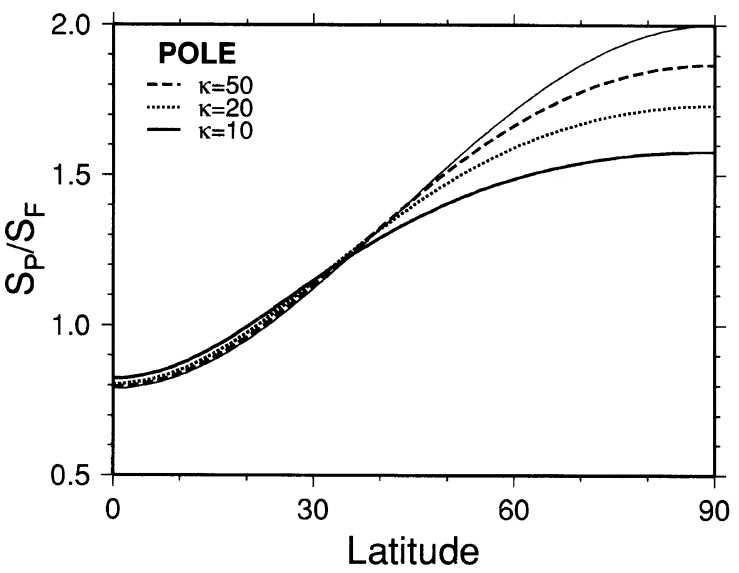

Fig. 2. Latitude variation of the ratio of ASD to that of original distribution for field directions mapped from Fisherian poles (a) and VGP positions mapped from Fisherian field directions (b). Theoretical curves by Cox (1970) are shown by a fine solid line. 
curve when $\kappa$ becomes larger, while the variation curve for field directions transferred from Fisherian poles does not show such tendency. Latitude variation curves of the ratio of ASD to that of the original distribution are shown in Fig. 2. There are also large discrepancies between simulated results and the theoretical curves in some cases, but the difference becomes smaller for larger $\kappa$. These errors are due to the fact that the formulation of Cox (1970) is derived by supposing that field directions which form a circular cone will be transferred to an oval of VGPs, which is an approximation for small radius of the cone. The larger discrepancy from theory for the smaller precision parameters observed throughout Figs. 1 and 2 are due to this fact.

\section{Paleosecular Variation from Lavas 3.1 Dataset from distinct sites}

Analysis of the Bingham statistics was applied to the database of PSV for the last 5 my by McElhinny and McFadden (1997), which includes all data in which the VGP latitude is higher than $45^{\circ}$. PSV data from each site did not show clear difference between the shape of the distribution of field directions and VGP positions. However, one exception was the data from Hawaii shown in Fig. 3 in which the equal area plot of field directions was made around the mean direction so that the shape of the distribution is easily seen (closed and open circles show normal and reversed polarities, respectively). The plot of field directions is similar to Hoffman (1984), but in the latter the center of the plot was the direction of the axial dipole field. Considering the difference angle of almost $9^{\circ}$ between the mean direction and the axial dipole field direction (or, far sidedness of $5^{\circ}$ for the mean pole) observed at Hawaii, it is better to plot field directions around the mean to see the shape of distribution. It is clearly observed that the distribution of field directions is elongated along the meridian plane while that of VGP positions are almost circular. This is also supported by the fact that $\alpha_{32} / \alpha_{31}$ of field directions is 1.49 with upper and lower confidence limits of 1.69 and 1.34, while that of VGPs is 1.18 with confidence limits of 1.33 and 1.08 . Here, the confidence limits were numerically determined by a bootstrap method (Efron and Gong, 1983). The elongation parameter of 1.49 for field directions at Hawaii is fairly close to the simulated value for the mean site latitude of $21^{\circ}$ as known from Fig. 1(a).

From these observations, it is suspected that the distribution of paleomagnetic directions observed at Hawaii is close to that of the field directions which are transferred from almost Fisherian poles. To ascertain this, histogram of angular distances from the mean was examined for both field and VGP to test whether they are Fisherian or not. Figure 4 shows the histograms with the theoretical distribution curves which correspond to the obtained precision parameters (28.27 and 44.52 for field and VGP, respectively). It is recognized that fitness of the histogram to the expected distribution curve seems to be high, especially for VGP. The observed distributions were compared to the Fisher distribution by $\chi^{2}$ test and Kolmogorov-Smirnov (K-S) test, following Chapter 14 of
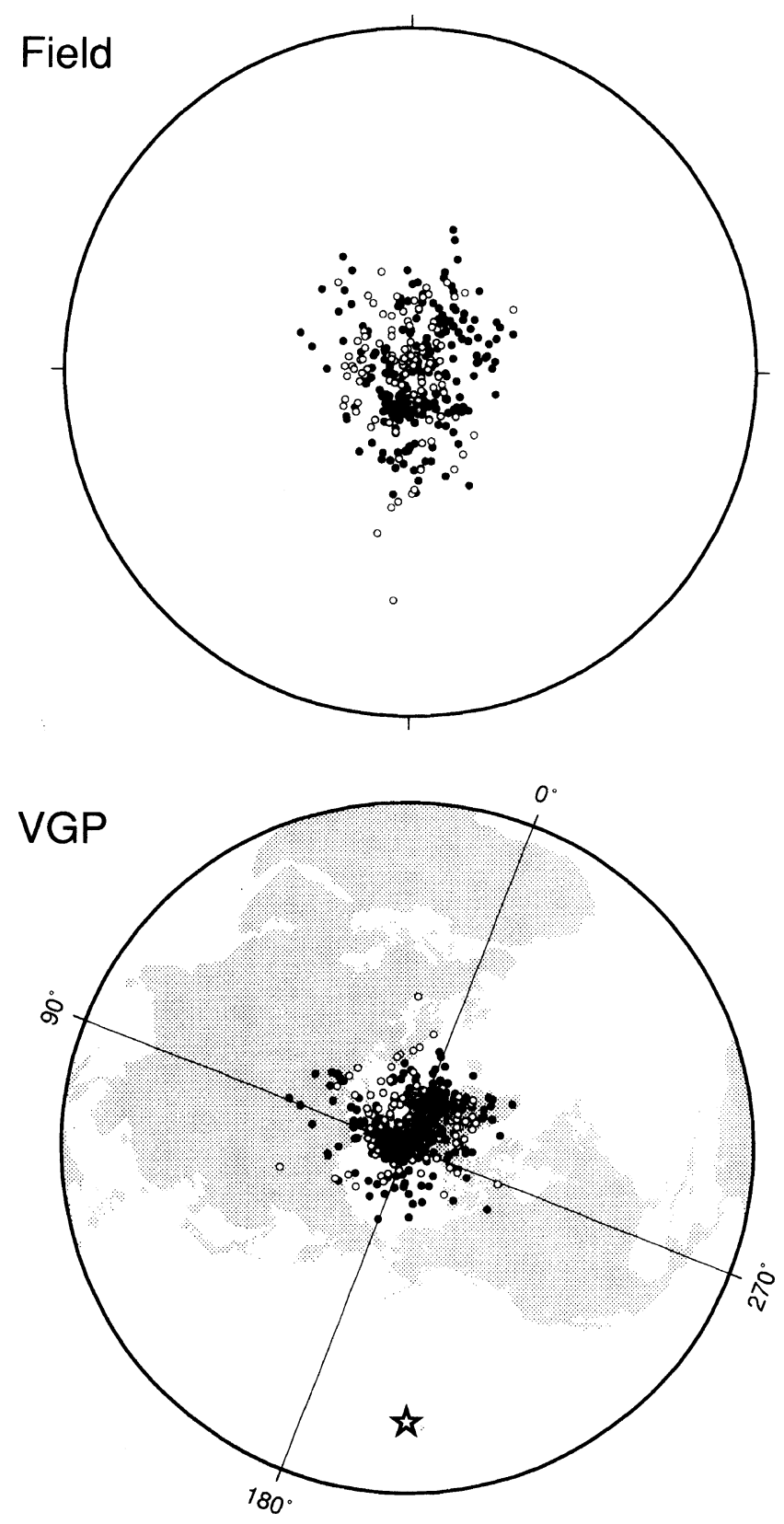

Fig. 3. Equal area plots of paleomagnetic directions around the mean (a) and VGP positions (b) from 324 lavas at Hawaii for the last 5 my. Elongation along the meridian plane is observed in field directions while the distribution of VGPs is almost circular. Closed and open circles denote normal and reversed polarities, respectively. Open star indicates the site locality of Hawaii which is the mean position of six sites.

Press et al. (1992). As the $\chi^{2}$ test is often sensitive to bin width, bins were divided so that each bin includes equal percentile of the theoretical distribution, and the $\chi^{2}$ test was repeated for the numbers of bins from 4 to 20. Both of $\chi^{2}$ test and K-S test indicate that the null hypothesis of the data distribution being Fisherian cannot be rejected for both field and VGP. Results of the statistical tests were summarized in Table 1 , in which only four results of $\chi^{2}$ test were shown because the results are more or less similar for other bin numbers. It is noted that the significance level is larger for VGP than for field in both results of $\chi^{2}$ and K-S tests, and this indicates the better 
Table 1. Statistical test to compare Hawaii data with Fisher distribution.

\begin{tabular}{rrrrrr}
\hline & \multicolumn{2}{c}{$\chi^{2}$ Test } & & \multicolumn{2}{c}{ K-S Test } \\
\cline { 2 - 3 } \cline { 5 - 6 }$N_{\text {bin }}$ & \multicolumn{1}{c}{$\chi^{2}$} & $p$ & & $D$ & $p$ \\
\hline Field & & & & \\
8 & 8.69 & 0.2756 & & 0.0538 & 0.2974 \\
12 & 7.70 & 0.7396 & & \\
16 & 13.88 & 0.5349 & & \\
20 & 16.99 & 0.5907 & & \\
& & & & \\
$V G P$ & & & & \\
8 & 2.52 & 0.9257 & & 0.0261 & 0.9787 \\
12 & 5.56 & 0.9013 & & \\
16 & 10.42 & 0.7925 & & \\
20 & 13.90 & 0.7894 & & \\
\hline
\end{tabular}

Note:

$N_{\text {bin }}$ : number of bins (only four results are shown although the test was repeated for 17 cases from 4 to 20$), \chi^{2}$ : observed $\chi^{2}$ statistic, $D$ : observed K-S statistic, $p$ : probability for $\chi^{2}$ or K-S statistic to exceed the observed value.

Fisher statistics of Hawaii data $(N=324)$ :

Field: $\bar{I}=28.9^{\circ}, \bar{D}=1.0^{\circ}, R=312.58, \kappa=28.27, \alpha_{95}=1.5^{\circ}$.

VGP: mean pole $\left(84.7^{\circ} \mathrm{N}, 11.9^{\circ} \mathrm{E}\right), R=316.74, \kappa=44.52, A_{95}=1.2^{\circ}$

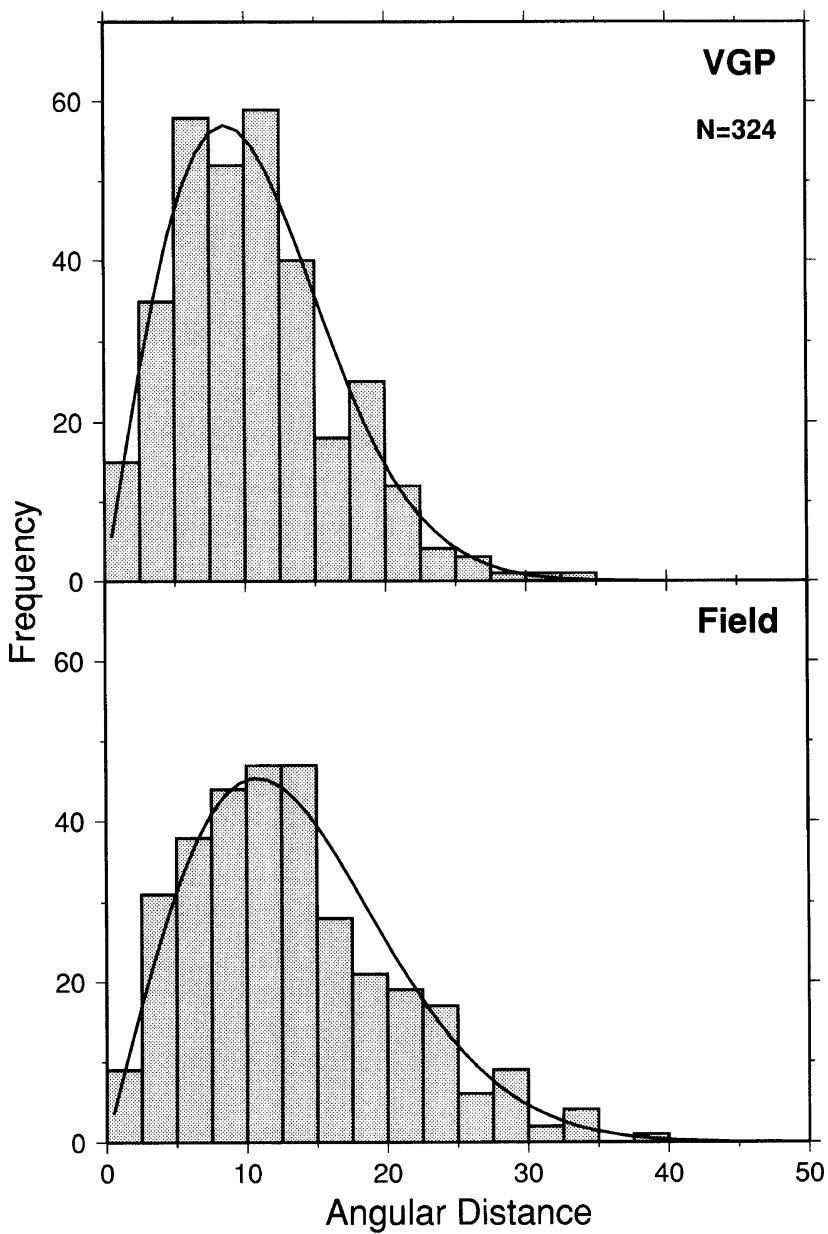

Fig. 4. Histograms of angular distances for VGP (upper figure) and paleodirections (lower figure), where the angular distances are measured from the mean. Solid curves indicate expected distribution from the Fisher distribution with the observed precision parameter (28.27 and 44.52 for field and VGP, respectively). fitness of the Fisher distribution to VGP positions than to field directions.

\subsection{Dataset from latitudinal bands}

As the analysis by the Bingham statistics to individual sites did not indicate statistically significant results except Hawaii, the same analysis was made to global data divided into latitudinal bands. Statistical parameters for the distribution of field directions were determined after transferring them to those in the axial dipole coordinate at each site so that the effect of difference in the latitude of site location within each band is compensated. Elongation of the distribution which was measured by $\alpha_{32} / \alpha_{31}$ increased at lower latitudinal bands, and the elongation was more
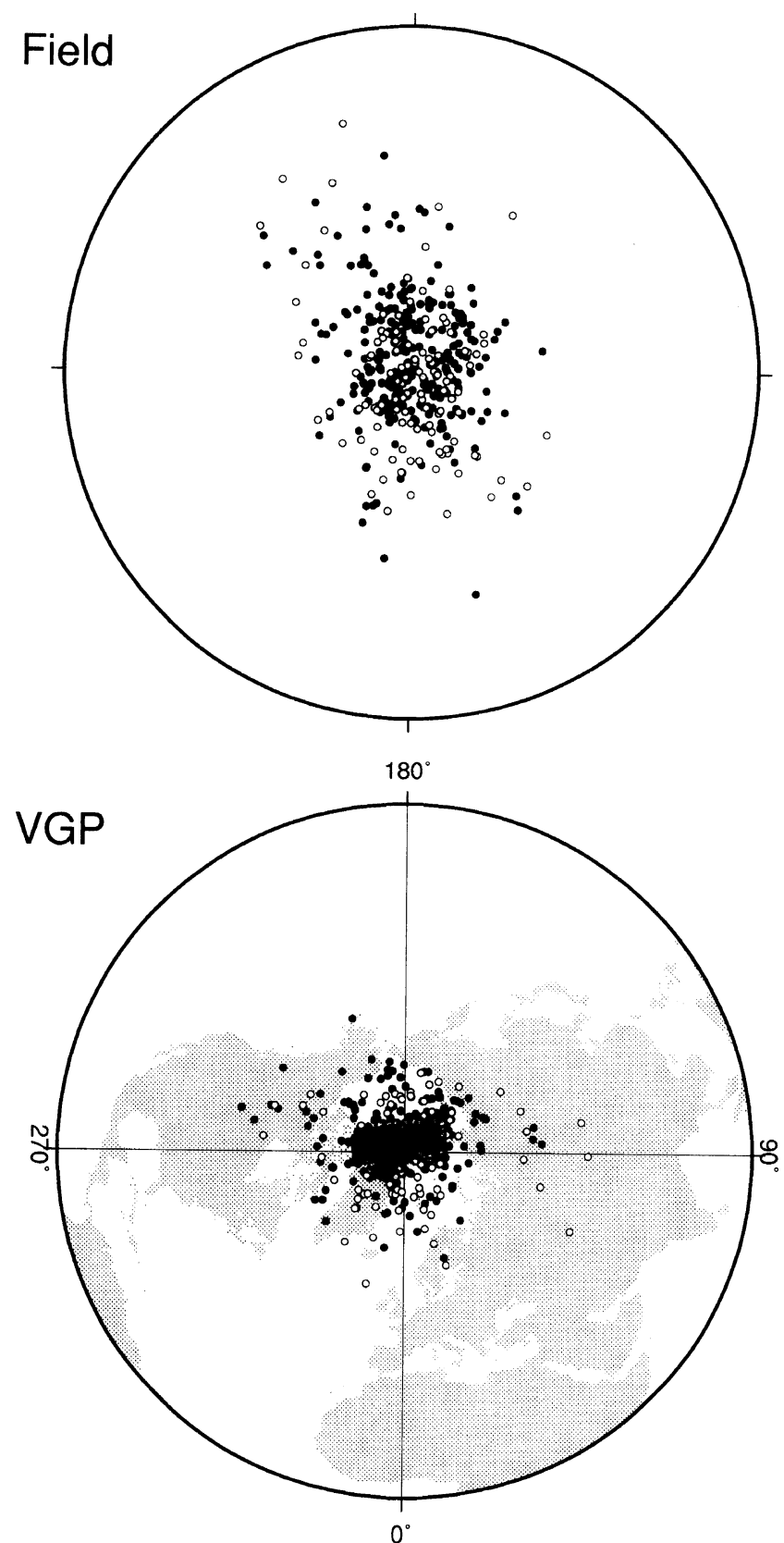

Fig. 5. Equal area plots of field directions (a) and VGPs (b) for the latitudinal band of $0^{\circ}-15^{\circ}$. The data were collected from 374 lavas at 16 sites for the last $5 \mathrm{my}$ and combined between the northern and the southern hemispheres. 
(a)
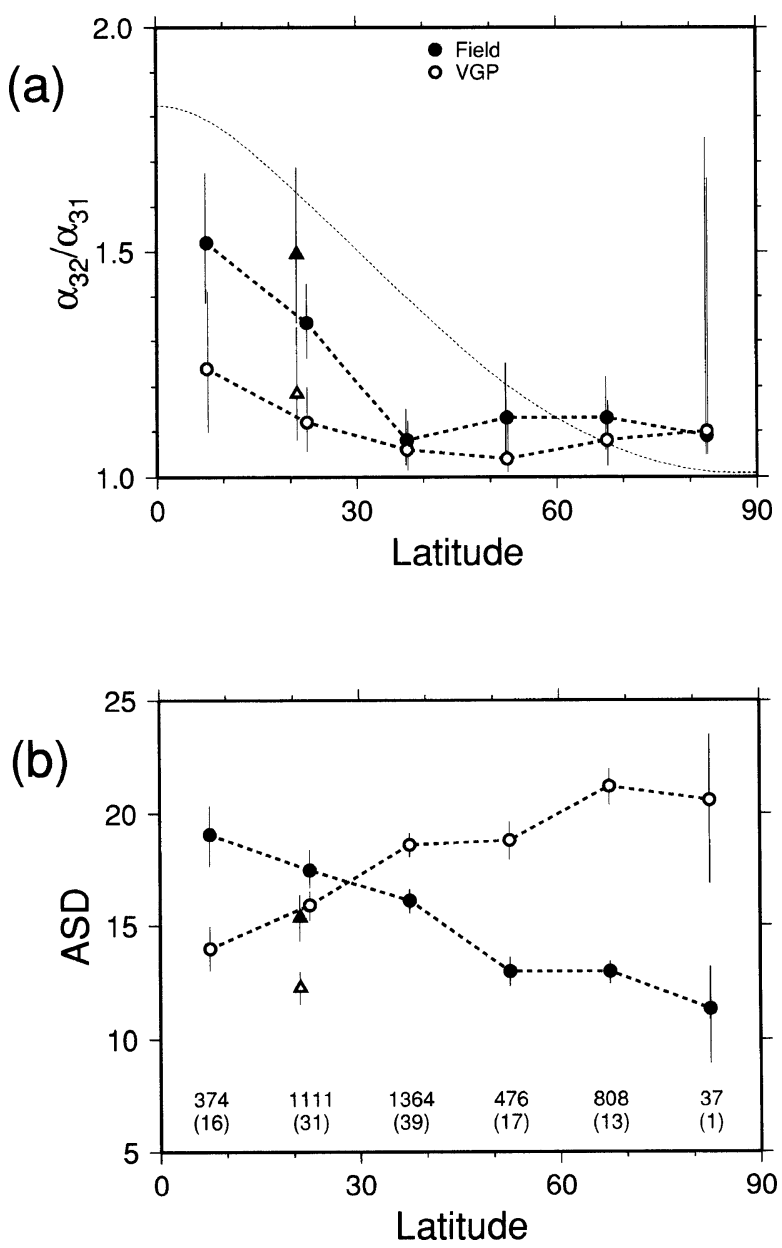

Fig. 6. Latitude variation of $\alpha_{32} / \alpha_{31}$ (a) and ASD (b) for the global data divided by latitudinal bands with the band width of $15^{\circ}$ and combined between the northern and the southern hemispheres. Solid and open circles denote those for field and VGP, respectively. Error bars indicate the confidence limits determined by a bootstrap method. Numbers of lavas are shown for each band, and those in the parentheses indicate the numbers of site location. Triangles indicate those from Hawaii data.

obvious in field than in VGP. Confidence limits of $\alpha_{32} / \alpha_{31}$ were determined by a bootstrap method, but they are often too large to conclude the significance of the difference between field and VGP, especially when the width of the latitudinal band is $10^{\circ}$ or less. However, the results become clearer when the analysis was made to the dataset which were combined between the northern and the southern hemispheres.

Equal area plots of field directions and VGP positions from 374 lavas for the band of $0^{\circ}-15^{\circ}$ are shown in Fig. 5 in which the difference of distribution is clearly seen. Similar tendency of the distribution is also seen for the combined band of $15^{\circ}-30^{\circ}$ and in the lower latitudinal bands when the width of the band is $20^{\circ}$. More quantitative representation is given in Fig. 6(a) by $\alpha_{32} / \alpha_{31}$ versus latitude when the band width is $15^{\circ}$. In the figure, the obtained values of $\alpha_{32} / \alpha_{31}$ from Hawaii are also shown by triangles, and the fine dotted line indicates the simulated curve for $\kappa=20$ which is more realistic to be compared than the theory of Cox (1970). The difference of $\alpha_{32} / \alpha_{31}$ between field and VGP is clearly observed in the lowest
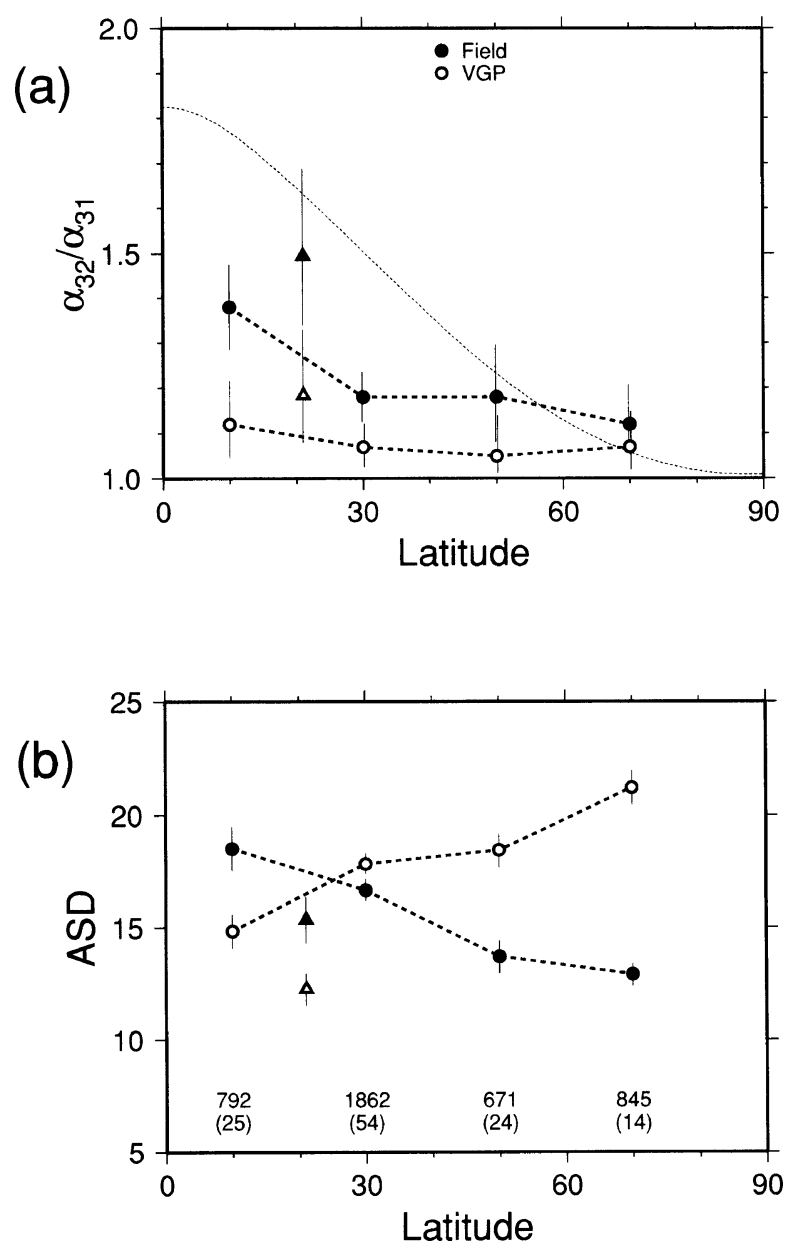

Fig. 7. Latitude variation of $\alpha_{32} / \alpha_{31}$ (a) and ASD (b) for the global data when the band width is $20^{\circ}$.

two latitudinal bands. The difference is significant for the band $15^{\circ}-30^{\circ}$, and possibly so for the band $0^{\circ}-15^{\circ}$ although small overlap is recognized between the bars of confidence limits. Confidence limits of $\alpha_{32} / \alpha_{31}$ are generally larger than those of ASD of which latitude variation is shown in Fig. 6(b), and this is an indication of less robustness in the determination of this parameter. Nevertheless, it is obvious that the distribution of field directions is more elongated than VGPs for the lower latitudinal bands. This becomes significant when the band width of $20^{\circ}$ is taken, as shown in Fig. 7, because the bars of confidence limits do not overlap for the two lowest bands, although the values of $\alpha_{32} / \alpha_{31}$ decrease compared to those for the case of band width of $15^{\circ}$.

\section{Discussion}

More elongation in field directions than poles at low latitude was shown from the global data divided by latitudinal bands. Although most of individual site did not reveal significant elongation, one notable result was obtained from Hawaii, in which the elongation parameter is as large as in the latitude band of $0^{\circ}-15^{\circ}$. Considering the latitude of Hawaii higher than the mean latitude of the lowest band, the elongated field directions at Hawaii is close to those transferred from Fisherian poles. This raises a supposition that the paleomagnetic field observed 
at Hawaii is mostly from a dipole wobble. This is supported by very high significance of the Fisher distribution to describe the observed VGP positions (Fig. 4 and Table 1), not to mention the small ASDs at Hawaii (Figs. 6(b) and 7(b)). This could be an indication of the Pacific Nondipole Low which has been long disputed on its real existence since Doell and Cox (1971). Even though the most recent review by McElhinny et al. (1996) concluded its rejection, this is still an important issue to pursue as the recent work by Shibuya et al. (1995) extended the idea to larger area of the Pacific region with low ASD versus the Atlantic region with high $\mathrm{ASD}$.

Elongated field directions observed at low latitude bands of the global data also suggest that the distribution of poles is more close to Fisherian than field directions, although the elongation is not as high as at Hawaii if the low latitude is taken into account. Besides, a null hypothesis of being Fisherian for the global data in the latitude bands of $0^{\circ}-15^{\circ}$ was rejected for both field and VGP with very

Table 2. Statistical test to compare the global data $\left(0^{\circ}-15^{\circ}\right)$ with Fisher distribution.

\begin{tabular}{rccccc}
\hline & \multicolumn{2}{c}{$\chi^{2}$ Test } & & \multicolumn{2}{c}{ K-S Test } \\
\cline { 2 - 3 } \cline { 5 - 6 }$N_{\text {bin }}$ & $\chi^{2}$ & $p$ & & $D$ & $p$ \\
\hline Field & & & & \\
8 & 31.82 & 0.0000 & & 0.1240 & 0.0000 \\
12 & 39.90 & 0.0000 & & \\
16 & 48.67 & 0.0000 & & \\
20 & 46.75 & 0.0004 & & \\
& & & & \\
$V G P$ & & & & \\
8 & 23.78 & 0.0012 & 0.1255 & 0.0000 \\
12 & 35.60 & 0.0002 & & \\
16 & 38.92 & 0.0007 & & \\
20 & 49.32 & 0.0002 & & \\
\hline
\end{tabular}

Note:

See the foot note of Table 1 for explanation of the notation. Fisher statistics of $0^{\circ}-15^{\circ}$ band data $(N=374)$ :

Field (axial dipole field coordinate): $\bar{I}=87.1^{\circ}, \bar{D}=354.1^{\circ}, R=$ $353.96, \kappa=18.61, \alpha_{95}=1.7^{\circ}$

VGP: mean pole $\left(88.0^{\circ} \mathrm{N}, 196.5^{\circ} \mathrm{E}\right), R=363.05, \kappa=34.05, A_{95}=1.3^{\circ}$. low significance level as summarized in Table 2. This is not surprising because even the largest dataset from Iceland is not Fisherian (Kristjansson and McDougall, 1982; Camps and Prévot, 1996). However, it is probably possible to interpret this feature of more elongation in field directions as a general indication of dipole nature which the paleomagnetic field contains. This observation is very natural from the fact that the paleomagnetic field is well approximated by a dipole field which has been established by various evidences such as success of the geocentric axial dipole hypothesis in paleomagnetism applied to plate movement (ex., McElhinny, 1973; Gordon, 1995), paleoinclination versus latitude (ex., Opdyke, 1972; Schneider and Kent, 1990), mean paleointensity versus paleolatitude (Tanaka et al., 1995; Perrin and Shcherbakov, 1997), and low-degree spherical harmonic analysis (ex., Creer et al., 1973; Johnson and Constable, 1997).

Although the elongation in the shape of distribution of field and VGP was not statistically significant at individual site, the directions of the principal axes were sensitive enough to reveal this feature. As the main eigenvector, $\boldsymbol{t}_{3}$, is always near vertical or close to the pole (here, field directions are plotted on the axial dipole field coordinate), other two eigenvectors lie near horizon or the equator. The $\boldsymbol{t}_{2}$ vector, the direction of the elongation, determined from field directions at each site, often clusters around up and down directions (or north and south directions). On the other hand, the $\boldsymbol{t}_{2}$ vector determined from VGP positions at each site often comes to the region with the longitude $\pm 90^{\circ}$ away from the site locality. Distribution of the directions of $\boldsymbol{t}_{2}$ vectors is shown in Fig. 8 as a sector diagram in which frequencies of azimuths of $\pm \boldsymbol{t}_{2}$ vectors were plotted. Preferred directions for field are obvious (a), while there is no clear feature for VGP (b). When the azimuth is expressed as a common-site longitude, preferred directions for VGP are remarkable (c). This is probably an indication that the anisotropy analysis is very sensitive to reveal the structure of the distribution, as often observed in the study of magnetic fabric.

The preferred directions in $\boldsymbol{t}_{2}$ vectors from the distribution of VGPs, viewed at a common-site longitude as shown in Fig. 8(c), are most probably due to the effect of mapping (a) Field

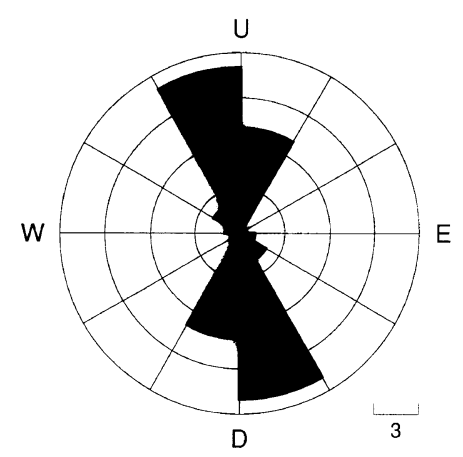

(b) VGP

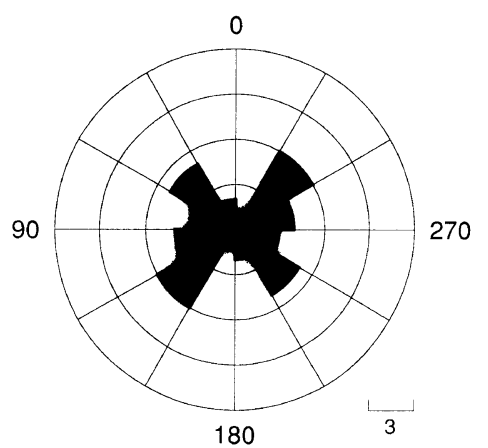

(c) VGP (common site)

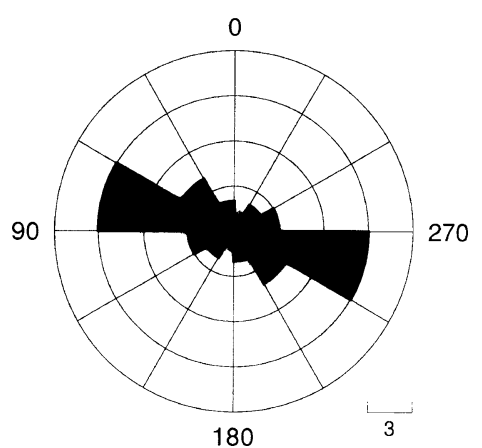

Fig. 8. Sector diagrams of the directions of $\pm \boldsymbol{t}_{2}$ eigenvectors, the directions of elongation, determined from separate dataset at individual site. Preferred directions are obvious in field directions (a) while there is no clear feature in VGPs (b). When the azimuth of the $\boldsymbol{t}_{2}$ vectors is represented by a common-site longitude, preferred directions become obvious in VGPs (c). 
from field directions. It is reasonable that the distribution of field directions includes more or less Fisherian component due to various reasons such as variations in nondipole terms or measurements error. This effect was predicted by Egbert (1992) who derived statistical representation of the densities for VGP longitude and pointed out some connection to the preferred transitional path which is still in dispute about its cause. On the other hand, the preferred directions in $\boldsymbol{t}_{2}$ vectors from the distribution of field directions can be interpreted as a result of mapping the more circular distribution of poles to the field directions elongated along the meridian plane. This is important because field directions are what we really observe and their elongated distribution is a true nature of the geomagnetic field.

\section{Conclusions}

Oval-shaped distribution of field directions was observed in the data from Hawaii for the last 5 my. Although other individual site did not give statistically significant results, the global data divided by latitudinal bands also showed more elongation in field directions at the low latitude bands. When $\alpha_{32} / \alpha_{31}$ was used as an elongation parameter, significant difference was observed between field and VGP at the lowest latitude band for the dataset combined between the northern and the southern hemispheres. This feature of paleomagnetic field was interpreted as a general indication of the dipole nature of the geomagnetic field. However, for the case of Hawaii, possibility of Pacific Nondipole Low was suggested because of the large elongation of the field directions and very high significance of the Fisher distribution to describe the observed distribution of VGPs, no mention to the small observed ASDs in field and VGP. Although the individual site did not give significant elongation in field directions, there are strong preferred directions in the principal axes determined by the Bingham analysis for both field directions and VGP positions, which are concordant with the general indication of the dipole nature. Even though the dipole nature is one of established features of the mean paleomagnetic field, it is still worthwhile to support this from different aspects.

Acknowledgments. I thank Leo Kristjansson of University of Iceland and Chris Harrison of University of Miami for many constructive review comments which greatly improved the manuscript.

\section{Appendix. Random Generator of the Bingham Distribution}

The rejection method described at Chapter 7.3 of Press et al. (1992) can be applied to generate random points $(\theta$, $\phi)$, which follow the Bingham distribution with the concentration parameters $k_{1}$ and $k_{2}$, over $0^{\circ} \leq \theta \leq 90^{\circ}$ and $0^{\circ} \leq \phi \leq 360^{\circ}$ by introducing the following probability density function (actually, a non-normalized Fisher distribution).

$$
f(\theta, \phi)=\frac{1}{4 \pi d\left(k_{1}, k_{2}\right)} e^{-k_{2}(\cos \theta-1)}
$$

$f(\theta, \phi)$ can be used as a comparison function in the rejection method because it is easily shown that,

$$
f(\theta, \phi) \geq b(\theta, \phi) \quad\left(0^{\circ} \leq \theta \leq 90^{\circ}\right) .
$$

More specifically, first generate random points which follow the Fisher distribution,

$$
\left\{\begin{array}{l}
\theta=\arccos \left(-\frac{1}{k_{2}} \log \left(2 \sinh \left(-k_{2}\right) X_{1}\right)\right) \\
\phi=2 \pi X_{2}
\end{array}\right.
$$

where $X_{1}$ and $X_{2}$ are random numbers between 0 and 1 . Then adopt or reject this point according to the following ratio,

$$
b(\theta, \phi) / f(\theta, \phi)=e^{\left(k_{1} \cos ^{2} \phi+k_{2} \sin ^{2} \phi\right) \sin ^{2} \theta+k_{2}(\cos \theta-1)} .
$$

\section{References}

Baag, C.-G. and C. E. Helsley, Shape analysis of paleosecular variation data, J. Geophys. Res., 79, 4923-4932, 1974.

Bingham, C., An antipodally symmetric distribution on the sphere, Ann. Stat., 2, 1201-1225, 1974.

Camps, P. and M. Prévot, A statistical model of the fluctuations in the geomagnetic field from paleosecular variation to reversal, Science, 273, 776-779, 1996

Cox, A., Latitude dependence of angular dispersion of the geomagnetic field, Geophys. J. R. astr. Soc., 20, 253-269, 1970

Creer, K. M., The dispersion of the geomagnetic field due to secular variation and its determination for remote times from paleomagnetic data, J. Geophys. Res., 67, 3461-3476, 1962.

Creer, K. M., E. Irving, and A. E. M. Nairn, The paleomagnetism of the Great Whin Sill, Geophys. J. R. astr. Soc., 2, 306-323, 1959.

Creer, K. M., D. T. Georgi, and W. Lowrie, On the representation of the Quaternary and Late Tertiary geomagnetic fields in terms of dipoles and quadrupoles, Geophys. J. R. astr. Soc., 33, 323-345, 1973.

Doell, R. R. and A. Cox, Pacific geomagnetic secular variation, Science, 171, 248-254, 1971.

Efron, B. and G. Gong, A leisurely look at the bootstrap, the jackknife, and cross-validation, Am. Stat., 37, 36-48, 1983.

Egbert, G. D., Sampling bias in VGP longitudes, Geophys. Res. Lett., 19, 2353-2356, 1992.

Fisher, R. A., Dispersion on a sphere, Proc. Roy. Soc., A217, 295-305, 1953.

Gordon, R. G., Plate motions, crustal and lithospheric mobility, and paleomagnetism: Prospective viewpoint, J. Geophys. Res., 100, 24367-24392, 1995.

Hoffman, K. A., A method for the display and analysis of transitional paleomagnetic data, J. Geophys. Res., 89, 6285-6292, 1984.

Johnson, C. L. and C. G. Constable, The time-averaged geomagnetic field: global and regional biases for 0-5 Ma, Geophys. J. Int., 131, 643-666, 1997.

Kristjansson, L. and I. McDougall, Some aspects of the late Tertiary geomagnetic field in Iceland, Geophys. J. R. astr. Soc., 68, 273-294, 1982.

McElhinny, M. W., Palaeomagnetism and Plate Tectonics, 358 pp., Cambridge University Press, Cambridge, 1973.

McElhinny, M. W. and P. L. McFadden, Palaeosecular variation over the past 5 Myr based on a new generalized database, Geophys. J. Int., 131, 240-252, 1997

McElhinny, M. W., P. L. McFadden, and R. T. Merrill, The myth of the Pacific dipole window, Earth Planet. Sci. Lett., 143, 13-22, 1996.

Onstott, T. C., Application of the Bingham distribution function in paleomagnetic studies, J. Geophys. Res., 85, 1500-1510, 1980. 
Opdyke, N. D., Paleomagnetism of deep-sea cores, Rev. Geophys. Space Phys., 10, 213-249, 1972.

Perrin, M. and V. Shcherbakov, Paleointensity of the earth's magnetic field for the past $400 \mathrm{Ma}$ : evidence for a dipole structure during the Mesozoic low, J. Geomag. Geoelectr., 49, 601-614, 1997.

Press, W. H., S. A. Teukolsky, W. T. Vetterling, and B. P. Flannery, Numerical Recipes in C: The Art of Scientific Computing, 2nd edition, 994 pp., Cambridge University Press, Cambridge, 1992.

Schneider, D. A. and D. V. Kent, The time-averaged paleomagnetic field, Rev. Geophys., 28, 71-96, 1990.

Shibuya, H., J. Cassidy, I. E. M. Smith, and T. Itaya, Paleomagnetism of young New Zealand basalts and longitudinal distribution of paleosecular variation, J. Geomag. Geoelectr., 47, 1011-1022, 1995.

Tanaka, H., M. Kono, and H. Uchimura, Some global features of paleointensity in geological time, Geophys. J. Int., 120, 97-102, 1995.

Tsunakawa, H., Geomagnetic secular variation during the Brunhes epoch inferred from the paleomagnetism and the last 200 years geomagnetic field, J. Geomag. Geoelectr., 40, 1365-1385, 1988.

H. Tanaka (e-mail: hidefumi@cc.kochi-u.ac.jp) 